\title{
Dietary plasmalogen increases erythrocyte membrane plasmalogen in rats
}

\author{
Shiro Mawatari ${ }^{1 *}$, Toshihiko Katafuchi ${ }^{2}$, Kiyotaka Miake ${ }^{3}$ and Takehiko Fujino ${ }^{4}$
}

\begin{abstract}
Background: Many disorders with plasmalogen deficiency have been reported. Replenishment or replacement of tissue plasmalogens of these disorders would be beneficial to the patients with these disorders, but effects of dietary plasmalogen on mammals have not been reported.

Methods: Plasmalogens were purified from chicken skin. The purified plasmalogens consisted of 96.4\% ethanolamine plasmalogen (PlsEtn), 2.4\% choline plasmalogen (PlsCho) and 0.5\% sphingomyelin (SM). A diet containing $0.1 \%$ the purified plasmalogens (PIsEtn diet) was given to rats. Relative composition of phospholipids was measured by a high performance liquid chromatography (HPLC) method that can separate intact plasmalogens and all other phospholipid classes by a single chromatographic run.

Results: The PlsEtn diet given to Zucker diabetic fatty (ZDF) rats for 4 weeks caused decreases of plasma cholesterol and plasma phospholipid as compared to control diet. The other routine laboratory tests of plasma including triacylglycerol, glucose, liver and renal functions, albumin, and body weight were not different. Relative compositions of erythrocyte PIsEtn and phosphatidylethanolamine (PE) increased, and that of phosphatidylcholine (PC) decreased in PlsEtn diet group. The PIsEtn diet given to normal rats for 9 weeks again caused decrease of plasma cholesterol and phospholipid, and it induced increase of relative composition of PlsEtn of the erythrocyte membrane. The other routine laboratory tests of plasma and body weight were not different.
\end{abstract}

Conclusions: Dietary PIsEtn increases relative composition of PlsEtn of erythrocyte membranes in normal and ZDF rats, and it causes decreases of plasma cholesterol and plasma phospholipids. Dietary PlsEtn for 9 weeks seemingly causes no adverse effect to health of normal rats.

Keywords: Dietary plasmalogen, Zucker diabetic fatty rat, Wistar rat, Erythrocyte phospholipids, Plasma phospholipids

\section{Background}

Plasmalogens are glycereophospholipids characterized by the presence of vinyl ether bond at the sn-1 position of glycerol backbone and an ester bond at the sn-2 position. The sn-1 position is most commonly linked to C16, C18, or C18:1 fatty alcohols and the sn-2 position typically occupied by an polyunsaturated fatty acid, specifically arachidonic acid (ARA) or docosahexaenoic acid (DHA) [1-5]. Plasmalogens are found in almost all mammalian tissues and constitute about $18 \%$ of the total phospholipids in cell membranes, with ethanolamine plasmalogen (PlsEtn) much more abundant than choline

\footnotetext{
* Correspondence: mawatari@rheology.po-jp.com

${ }^{1}$ Institute of Rheological Function of Food, 2241 Kubara, Hisayama-chou, Kasuya-gun, Fukuoka 811-2501, Japan

Full list of author information is available at the end of the article
}

plasmalogen (PlsCho) except heart and skeletal muscle [1-5]. Plasmalogens are not only structural component of mammalian cell membrane and a reservoir for second messengers, but also may involve membrane fusion, ion transport and cholesterol efflux [1-5]. The vinyl ether bond at the sn-1 position makes plasmalogens more susceptible to oxidative stress than the corresponding ester bonded glycerophospholipids, plasmalogens may also act as antioxidants, protecting cells from oxidative stress [1-5].

Plasmalogen biosynthesis starts in peroxisome, and the inherited human peroxisomal disorder, rhizomelic chondrodysplasia punctata (RCDP), shows deficiency of tissue plasmalogens in multiple organs and causes severe disorders of multiple organs such as bone, brain, lens, kidney and heart [2-5]. These disorders of RCDP may be a 
direct consequence of plasmalogen deficiency and indicate importance of plasmalogens in human body. On the other hand, secondary plasmalogen deficiency was reported in metabolic and inflammatory disorders such as diabetes mellitus, cardiac diseases, cancer, respiratory diseases and Alzheimer's disease [3,4]. Secondary plasmalogen deficiency could result from decreased synthesis and/or increased degradation of plasmalogens. Replenishment and/or replacement of plasmalogens would be of substantial benefit in these diseases with plasmalogen deficiency.

Health effects of dietary phospholipids are recently reviewed [6], and the majority of studies indicated that dietary phospholipids have a positive impact in several diseases without severe side effect, however, dietary plasmalogen was not mentioned [6].

We prepared purified plasmalogens from chicken skin. We administered a diet containing $0.1 \%$ the purified plasmalogens to Zucker diabetic fatty (ZDF) rats and normal rats of Wistar strain. Routine laboratory tests of blood plasma (including total phospholipid) were done and phospholipid composition of plasma and erythrocytes were analyzed.

\section{Results and discussion}

The purified plasmalogens from chicken skin consisted of $96.4 \%$ ethanolamine plasmalogen (PlsEtn), 2.6\% choline plasmalogen (PlsCho), $0.5 \% \mathrm{SM}$ and $0.7 \%$ other phospholipids by calculation on the HPLC chromatographic area (Table 1, Figure 1). The retention times of chromatographic peaks of PlsEtn and PlsCho were accorded to those of PlsEtn and PlsCho of the rat erythrocyte membrane (Figure 1), and the peaks disappeared after treatment with hydrochloric acid $(\mathrm{HCl})$, indicating the peaks were plasmalogens $[7,8]$ (data are not shown). The fatty acids of the PlsEtn composed of DHA (22:6), ARA (20:4), oleic acid (18:1), myristic acid (14:0),

Table 1 Phospholipid composition of the purified plasmalogens and fatty acid composition of PIsEtn in the purified plasmalogens

\begin{tabular}{lccc}
\hline \multicolumn{2}{l}{ Phospholipid composition } & \multicolumn{2}{c}{ Fatty acid composition of PlsEtn } \\
\hline PlsEtn & Fatty acid \\
\hline PlsCho & $96.4 \%$ & $14: 0$ & $5.4 \%$ \\
\hline SM & $2.6 \%$ & $16: 0$ & $7.1 \%$ \\
\hline LPE & $0.5 \%$ & $18: 0$ & $3.5 \%$ \\
\hline & $0.7 \%$ & 18.1 & $35.4 \%$ \\
\hline & & $18: 2$ & $9.5 \%$ \\
\hline & $20: 4$ & $32.7 \%$ \\
\hline
\end{tabular}

Abbreviations; PIsEtn, ethanolamine plasmalogen; PlsCho, choline plasmalogen; SM, sphingomyelin: LPE, lysophosphatidylethanolamine. palmitic acid (16:0) and stearic acid (18:0) (Table 1), which is in agree with that of ordinary plasmalogens found in mammalian tissues [1-4].

The chromatogram of phospholipid analysis of the PlsEtn diet showed a large peak of PlsEtn as compared to the control diet, indicating that PlsEtn was actually contained in the PlsEtn diet (Figure 2).

The diet contained 0.1 weight $\%$ the purified plasmalogens (PlsEtn diet) caused a decrease of plasma phospholipids as well as plasma cholesterol in ZDF rats within 4 weeks (Table 2). However, the other routine laboratory tests of plasma including triacylglycerol (TG), glucose, alanine aminotransferase (ALT), aspartate aminotransferase (AST), urea nitrogen and albumin were not different (Table 2). Body weight was also not different in the both diet groups. Phospholipid composition of the plasma analyzed by our HPLC method showed an increase of SM in the PlsEtn diet group (Table 3). Plasmalogens in the plasma was detected by our HPLC method, but they were too small to evaluate changes of plasmalogens (Table 3, Figure 3). The PlsEtn diet induced increases of erythrocyte PlsEtn and PE, and decrease of erythrocyte PC (Table 4).

The same PlsEtn diet was administered to normal rats of Wistar strain for 9 weeks. The PlsEtn diet again caused decrease of plasma phospholipid as well as plasma cholesterol, but the other routine laboratory tests of plasma were not changed (Table 5). Phospholipid composition of plasma was not different (Table 6). Phospholipid composition of the erythrocyte membranes showed an increase of PlsEtn and tendency to decrease of PC in the PlsEtn diet group (Table 7).

These results show that dietary PlsEtn induces decrease of plasma cholesterol and plasma phospholipids in normal rats as well as in ZDF rats. It has been known that dietary phospholipids cause decrease of plasma cholesterol probably due to inhibition of intestinal cholesterol absorption $[6,9,10]$. There is a report that dietary PE, but not dietary PC, caused a decreases of phospholipid, cholesterol and apolipoprotein A-1 in rat blood plasama [11]. Thus it is indicated that ethanolamine phospholipids, not limited to PlsEtn, induce a decrease of plasma phospholipid. Phospholipid composition of plasma in normal rats (Wistar rats) was not changed after 9 weeks with the PlsEtn diet (Table 6), and the other routine laboratory tests in plasma were not changed (Table 5). Body weight was also not different after 9 weeks of the PlsEtn diet (Table 5). These results indicate that dietary PlsEtn is not bad for health of normal rats despite the decrease of plasma phospholipids and cholesterol. It may be interesting to note that plasma triacylglycerol was not changed by the PlsEtn diet in both ZDF rats and Wister rats despite the decrease of cholesterol and phospholipid. 

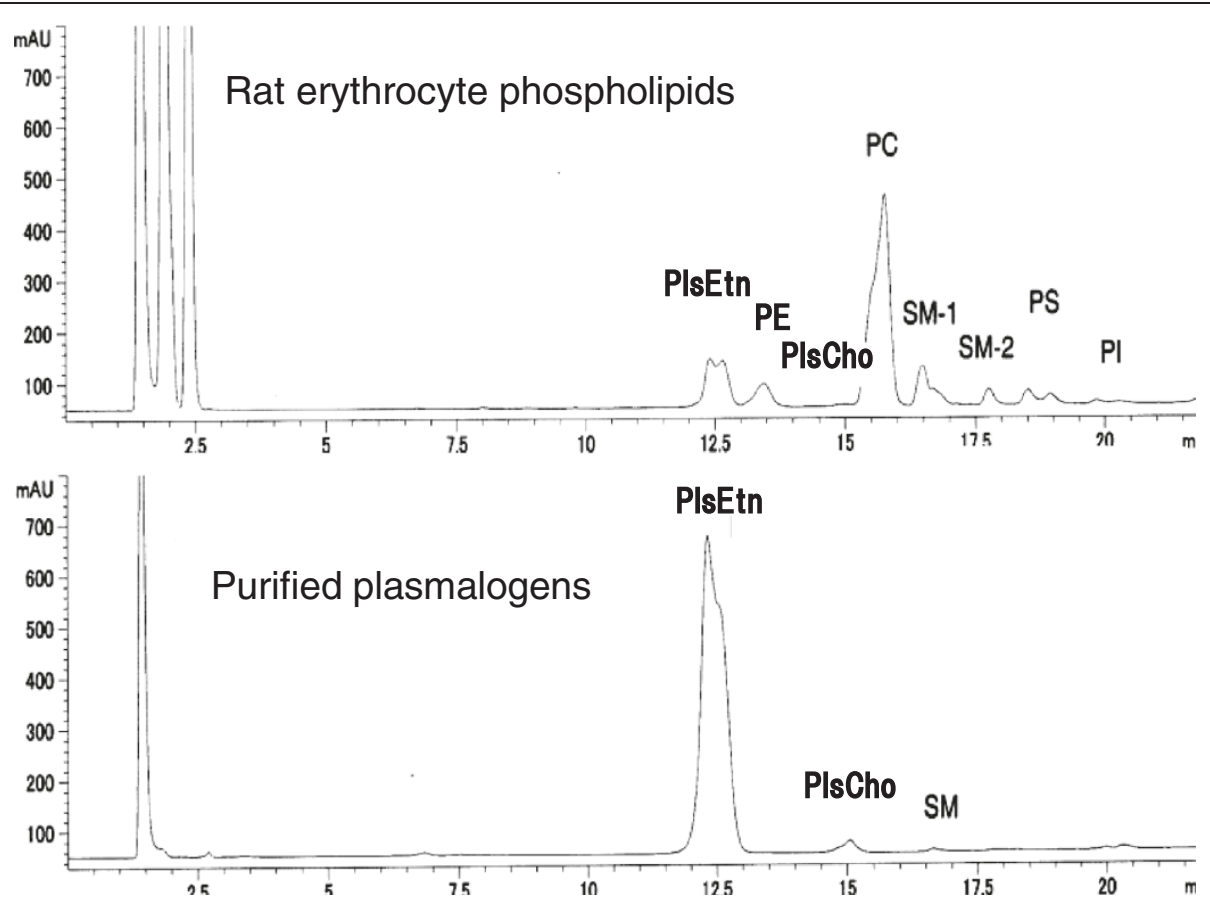

Figure 1 HPLC chromatogram of the purified plasmalogen from chicken skin. The retention times of ethanolamine plasmalogens (PIsEtn) and choline plasmalogen (PIsCho) are accorded to those of rat erythrocyte. The other abbreviations: PE, phosphatidylethanolamine; PC phosphatidylcholine; SM-1 and SM-2, sphingomyelin; PS, phosphatidyl serine; Pl, phosphatidyl inositol.

The PlsEtn diet to ZDF rats for 4 weeks induced increases of PlsEtn and PE, and decrease of PC in the erythrocyte membranes (Table 4). The PlsEtn diet to Wistar rats for 9 weeks also induced increase of PlsEtn and tendency to decrease of $\mathrm{PC}$ in the erythrocyte membrane (Table 7). Fatty acid composition of PlsEtn + PE showed an increase of ARA (20:4) and decrease of oleic acid (18:1) (Table 8). The increase of ARA in the erythrocyte membrane PlsEtn may reflect the fatty acid composition of PlsEtn in the diet (Table 1). These results
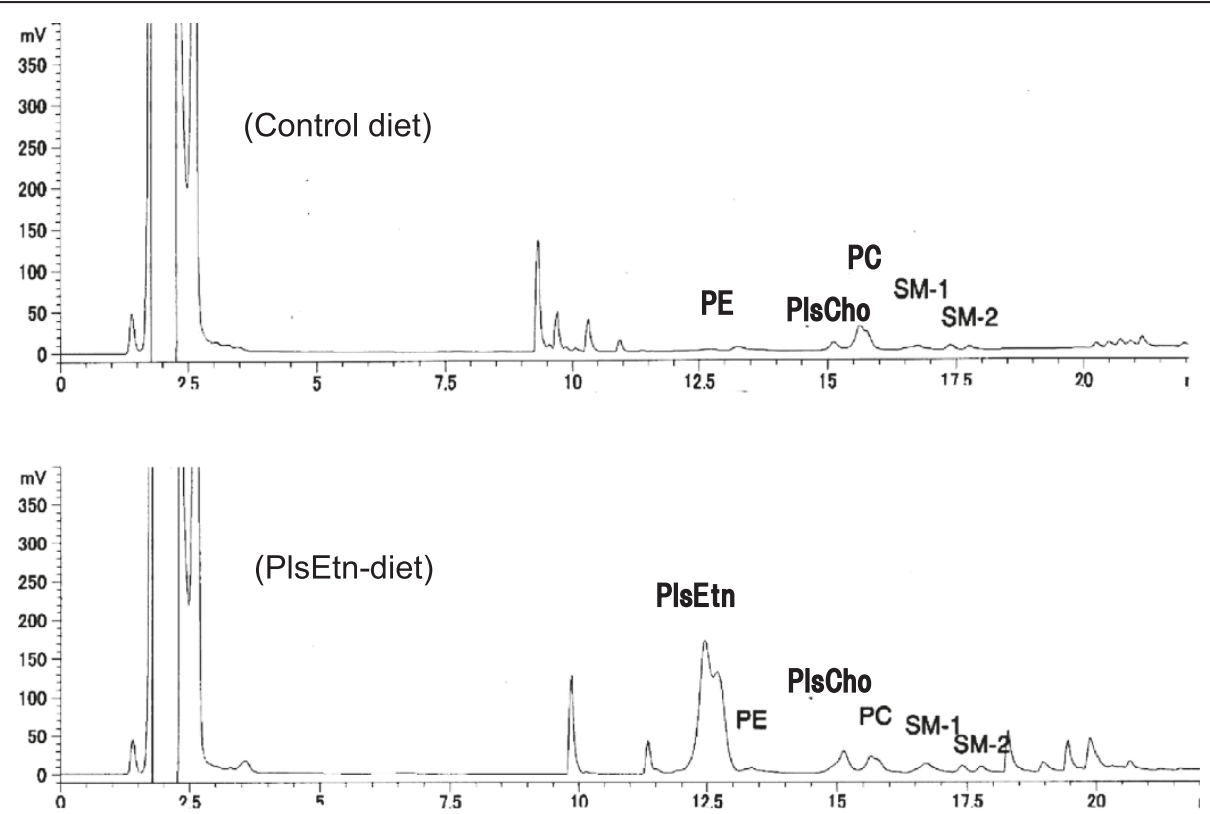

Figure 2 HPLC chromatograms of phospholipids of the control diet and the plasmalogen diet (PIsEtn diet). The chromatogram indicate that plasmalogens (PIsEtn and PIsCho) was actually contained in the PlsEtn diet. The other abbreviations: see Figure 1. 
Table 2 Changes in plasma cholesterol and phospholipids of ZDF rats after the PIsEtn for 4 weeks

\begin{tabular}{lccc}
\hline & $\begin{array}{c}\text { Control diet } \\
(\mathbf{n}=\mathbf{1 0})\end{array}$ & $\begin{array}{c}\text { PlsEtn diet } \\
(\mathbf{n}=\mathbf{1 0})\end{array}$ & t-test \\
\hline Total chol $(\mathrm{mg} / 100 \mathrm{~mL})$ & $206.3 \pm 17.4$ & $173.5 \pm 15.3$ & $p<0.001$ \\
\hline HDL-chol $(\mathrm{mg} / 100 \mathrm{~mL})$ & $60.3 \pm 14.1$ & $57.5 \pm 9.6$ & \\
\hline LDL-chol $(\mathrm{mg} / 100 \mathrm{~mL})$ & $9.2 \pm 4.4$ & $5.1 \pm 2.4$ & $p<0.02$ \\
\hline TG $(\mathrm{mg} / 100 \mathrm{~mL})$ & $1267.4 \pm 367.9$ & $1244.6 \pm 361.2$ & \\
\hline P-lipid $(\mathrm{mg} / 100 \mathrm{~mL})$ & $466.5 \pm 35.5$ & $409.3 \pm 51.1$ & $p<0.01$ \\
\hline Glucose $(\mathrm{mg} / 100 \mathrm{~mL})$ & $541.9 \pm 172.8$ & $507.5 \pm 155.2$ & \\
\hline Urea N $(\mathrm{mg} / 100 \mathrm{~mL})$ & $19.6 \pm 1.6$ & $19.0 \pm 2.1$ & \\
\hline AST $(\mathrm{IU} / \mathrm{L})$ & $135.6 \pm 94.2$ & $126.5 \pm 114.8$ & \\
\hline ALT $(\mathrm{IU} / \mathrm{L})$ & $96.1 \pm 57.7$ & $88.3 \pm 83.9$ & \\
\hline Albumin $(\mathrm{g} / 100 \mathrm{~mL})$ & $4.6 \pm 0.3$ & $4.5 \pm 0.3$ & \\
\hline Body weightt $(\mathrm{g})$ & $407.5 \pm 30.9$ & $391.5 \pm 40.1$ & \\
\hline Abbrans: & &
\end{tabular}

Abbreviations: chol, cholesterol; HDL, high density lipoprotein; LDL, low density lipoprotein; TG, triacylglycerol; P-lipid, phospholipid; Urea N, urea nitrogen; AST, aspartate aminotransferase; ALT, alanine aminotransferase. Data are mean $\pm S D$

of the erythrocyte membranes indicate that dietary PlsEtn can increase relative concentration of tissue PlsEtn.

Most of dietary glycerophospholipids may be hydrolyzed at the sn-2 position by pancreatic phospholipase A2 in the intestinal lumen and then absorbed by the enterocytes as free fatty acids and lysophospholipids $[6,12]$. The presence of plasmalogen-active phospholipase A2 in the small intestinal epithelium has been reported [13]. Because plasmalogens contain vinyl ether bond at the sn-1 position of glycerol, the lysophospholipid derived from dietary plasmalogens after digestion by phospholipase A2 is indicated to contain the vinyl ether bond. It is known that dietary alkyl glycerols are absorbed intact from the digestive tract without the cleavage of the ether bond [14-18]. However, whether the vinyl ether bond of dietary phospholipids absorbed intact from the intestine is not certain. The vinyl ether bond (1-0 alk-1'-enyl-sn-glycerol) is not same to ether

Table 3 Plasma phospholipid composition of ZDF rats after the PlsEtn diet for 4 weeks

\begin{tabular}{llll}
\hline & Control diet $(\mathbf{n}=\mathbf{1 0})$ & PlsEtn diet $(\mathbf{n}=\mathbf{1 0})$ & t-test \\
\hline PlsEtn & $0.39 \pm 0.31$ & $0.56 \pm 0.26$ & \\
\hline PE & $0.46 \pm 0.10$ & $0.63 \pm 0.22$ & \\
\hline PlsCho & $0.23 \pm 0.11$ & $0.35 \pm 0.10$ & \\
\hline PC & $87.66 \pm 1.14$ & $86.88 \pm 1.00$ & $p<0.01$ \\
\hline PI & $2.53 \pm 0.25$ & $2.55 \pm 0.23$ & \\
\hline SM & $2.08 \pm 0.47$ & $2.67 \pm 0.40$ & \\
\hline LPC & $5.60 \pm 0.77$ & $5.40 \pm 0.84$ &
\end{tabular}

Data are \% of total phospholipids calculated from chromatographic area detected by ELSD (mean \pm SD). bond of alkyl glycerols (1-0-alkyl-sn-glycerol). The vinyl ether bond is known to be especially sensitive to $\mathrm{HCl}$ $[7,8]$. There is a report, by using a diet containing $10 \%$ bovine brain phospholipids, that no degradation of plasmalogens under in vitro conditions simulating those of the stomach and small intestinal lumen was observed [19], and they also reported that ingestion of $10 \%$ bovine brain phospholipids increased plasmalogen in blood plasma [19]. Thus it is likely that lysophospholipids (lysoplasmalogens) containing the vinyl ether bond derived from the dietary PlsEtn are absorbed intact from rat intestine.

Since 1-0-alkylglycerol enter the plasmalogen biosynthesis pathway downstream of the peroxisomal step, plasmalogen replacement therapy for inherited peroxisomal disorders by using dietary 1-0 alkylglycerols are reported and the therapy induced increases of plasmalogens in several tissues [15-18,20]. Wood et al. [20,21] synthesized an alkyl-diacyl plasmalogen precursor with palmitic acid at sn-1, DHA at sn-2 and lipoic acid at sn3 (PPI-1011). They reported that PPI-1011 replenished PlsEtn of cultured lymphocytes from RCDP patients [20], and the oral administration of PPI-1011 to rabbits for 2 weeks increased DHA and DHA-containing PlsEtn in plasma and retina [21].

The PlsEtn diet to ZDF rats increased relative composition of PlsEtn of erythrocyte membranes within 4 weeks, and the same diet to normal rats for 9 weeks also increased relative composition of PlsEtn of the erythrocytes. The present study indicates that dietary PlsEtn is safe for health of normal rats despite the decrease of plasma phospholipids. Oral administration of purified plasmalogens may be more efficient for replenishment and/or replacement of plasmalogens of tissues than that of alkyl glycerols or alkyl-diacyl plasmalogen precursor (PPI-1011), because administration of plasmalogen may supply glycerol with vinyl ether bond, ethanolamine, choline and polyunsaturated fatty acids such as DHA and ARA, all of these substances may be re-used to biosynthesis of plasmalogens at various tissues.

Recently, we reported that pre-treatment with the purified plasmalogen from chicken breast muscle (composed $47.6 \%$ PlsEtn and $49.3 \%$ PlsCho) by intraperitoneal administration attenuate lipopolysaccharide-induced neuroinflammation [22]. However, whether dietary plasmalogens increase plasmalogen content of brain with plasmalogen deficiency remain to be tested.

\section{Materials and methods}

\section{Preparation of purified plasmalogens}

Plasmalogens were prepared from chicken skin. The chicken skin was obtained from a market place. Most of fat of the chicken skin was initially removed by a steam oven, then, the skin was freeze dried. Plasmalogens were 

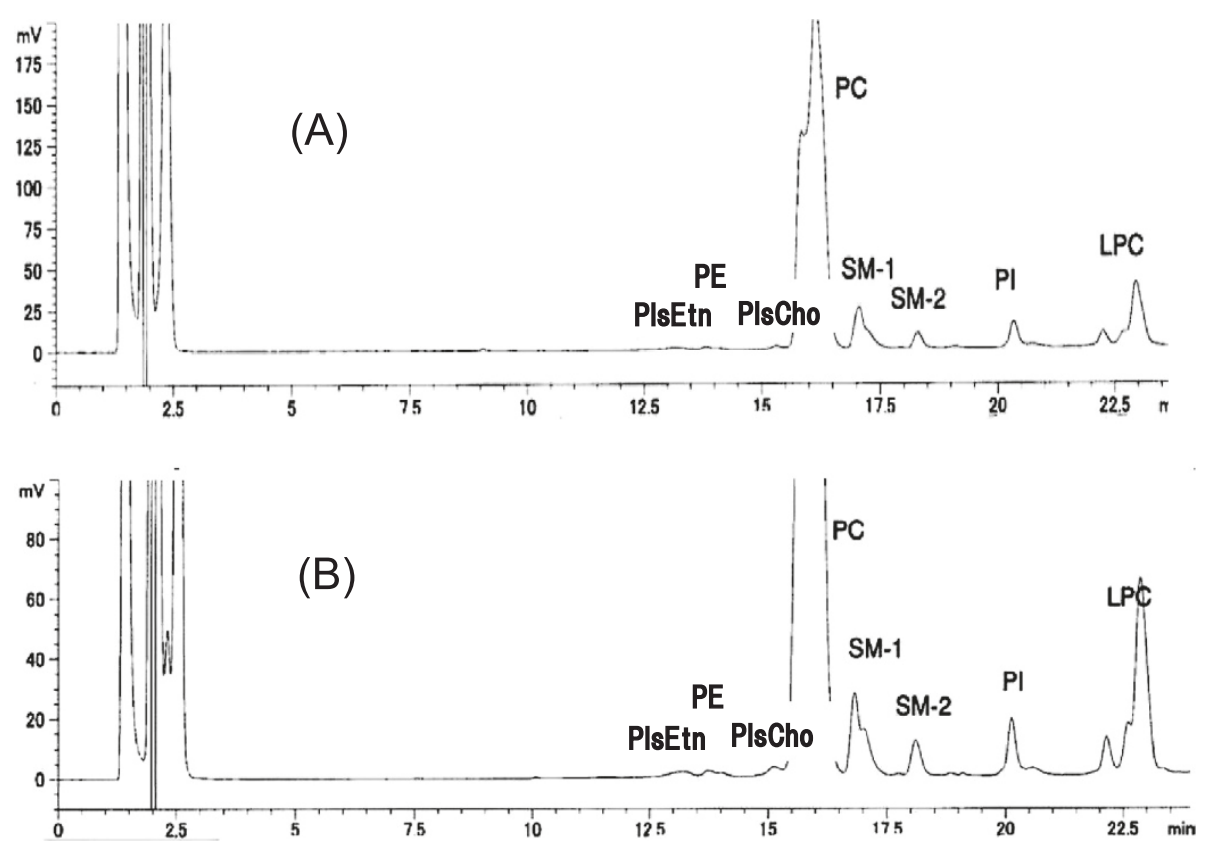

Figure 3 A representative HPLC chromatogram of plasma phospholipids in Wistar rat. Plasmalogens (PlsEtn and PlsCho) in rat plasma were detected by our method $(\mathbf{A})$, but they were too small to evaluate the changes of these plasmalogens even though chromatograph was magnified (B). LPC, lysophosphatidylcholine; the other abbreviations, see Figure 1.

purified from the freeze dried skin essentially by the procedure as reported previously [23].

\section{Animals and diets}

Diet containing 0.1 weight \% purified plasmalogens (PlsEtn diet) was prepared by supplementation of the purified plasmalogens to control diet (AIN 96). These diets were prepared and supplied by Oriental Yeast Co (Tokyo, Japan).

All of the experiments involving the use of animals were accorded to the Guiding Principles for the Care and Use of Animals of the Physiological Society of Japan, and all efforts were made to minimize animal suffering and the number of animals used for the experiments.

Table 4 Changes in erythrocyte phospholipid composition of ZDF rats after the PIsEtn diet for 4 weeks

\begin{tabular}{lccc}
\hline & Control diet $(\mathbf{n}=\mathbf{1 0})$ & PlsEtn diet $(\mathbf{n}=\mathbf{1 0})$ & t-test \\
\hline PlsEtn & $14.58 \pm 0.76$ & $15.65 \pm 0.75$ & $p<0.006$ \\
\hline PE & $7.94 \pm 0.54$ & $8.82 \pm 0.58$ & $p<0.003$ \\
\hline PlsCho & $0.39 \pm 0.08$ & $0.43 \pm 0.07$ & \\
\hline PC & $62.69 \pm 2.76$ & $59.74 \pm 1.53$ & $P<0.001$ \\
\hline PS & $4.21 \pm 1.04$ & $4.66 \pm 0.47$ & \\
\hline PI & $0.91 \pm 0.21$ & $1.04 \pm 0.15$ & \\
\hline SM & $9.27 \pm 0.63$ & $9.66 \pm 0.52$ & \\
\hline
\end{tabular}

Data are \% of total phospholipids calculated from chromatographic area detected by ELSD (mean \pm SD).
All rats were obtained from KBT Oriental Co. (Tosu City, Saga Prefecture, Japan). Twenty male rats of ZDF rats, aged 4 weeks, received the control diet for 10 days, and they were divided into two groups at random. One group (10 rats) was fed the control diet for an additional 4 weeks (control diet group), and the other groups (10 rats) was fed the PlsEtn diet (PlsEtn diet group) for 4 weeks. In another experiment, 18 male rats of Wistar

Table 5 Changes in plasma cholesterol and phospholipid of Wistar rats after the PlsEtn diet for 9 weeks

\begin{tabular}{|c|c|c|c|}
\hline & $\begin{array}{l}\text { Control diet } \\
\quad(n=8)\end{array}$ & $\begin{array}{l}\text { PlsEtn- diet } \\
\quad(n=8)\end{array}$ & t-test \\
\hline Total chol (mg/100mL) & $98.4 \pm 15.6$ & $81.1 \pm 10.2$ & $p<0.05$ \\
\hline HDL-chol (mg/100mL) & $53.6 \pm 11.3$ & $43.9 \pm 7.8$ & $p<0.07$ \\
\hline LDL-chol (mg/100mL) & $22.1 \pm 7.3$ & $16.6 \pm 5.5$ & $p<0.10$ \\
\hline $\mathrm{TG}(\mathrm{mg} / 100 \mathrm{~mL})$ & $145.1 \pm 76.7$ & $156.5 \pm 73.7$ & \\
\hline P-lipid (mg/100mL) & $183.3 \pm 22.9$ & $152.7 \pm 17.3$ & $p<0.01$ \\
\hline Glucose (mg/100mL) & $232.7 \pm 36.8$ & $220.9 \pm 36.7$ & \\
\hline Urea N (mg/100mL) & $12.9 \pm 1.9$ & $13.1 \pm 1.3$ & \\
\hline AST (IU/L) & $51.8 \pm 4.3$ & $53.4 \pm 4.9$ & \\
\hline $\mathrm{ALT}(\mathrm{IU} / \mathrm{L})$ & $24.9 \pm 3.6$ & $23.3 \pm 3.7$ & \\
\hline Albumin (g/100mL) & $4.4 \pm 0.2$ & $4.3 \pm 0.4$ & \\
\hline Body weight (g) & $478.8 \pm 19.1$ & $453.6 \pm 21.5$ & \\
\hline
\end{tabular}

Abbreviations: chol, cholesterol; HDL, high density lipoprotein; LDL, low density lipoprotein; TG, triacylglycerol; P-lipid, phospholipid; Urea N, urea nitrogen; AST, aspartate aminotransferase; ALT, alanine aminotransferase. Data are mean $\pm S D$. 
Table 6 Plasma phospholipid composition of Wistar rats after the PIsEtn diet for 9 weeks

\begin{tabular}{lcc}
\hline & Control diet $(\mathbf{n}=\mathbf{8})$ & PlsEtn diet $(\mathbf{n}=\mathbf{8})$ \\
\hline PlsEtn & $0.36 \pm 0.18$ & $0.41 \pm 0.23$ \\
\hline $\mathrm{PE}$ & $0.34 \pm 0.15$ & $0.38 \pm 0.17$ \\
\hline $\mathrm{PlsCho}$ & $0.43 \pm 0.11$ & $0.39 \pm 0.05$ \\
\hline $\mathrm{PC}$ & $80.11 \pm 1.24$ & $79.95 \pm 1.49$ \\
\hline $\mathrm{SM}$ & $5.78 \pm 0.81$ & $6.46 \pm 1.65$ \\
\hline $\mathrm{PI}$ & $3.03 \pm 0.29$ & $2.63 \pm 0.33$ \\
\hline $\mathrm{LPC}$ & $9.89 \pm 0.84$ & $9.73 \pm 1.11$ \\
\hline
\end{tabular}

Data are \% of total phospholipids calculated from chromatographic area detected by ELSD (mean \pm SD).

strain, aged 6 weeks, received the control diet for 10 days, and divided into two groups. One group received the control diet for additional 9 weeks (control diet group) and the other group received the PlsEtn diet for 9 weeks. All rats were housed in stainless steel wire cages in a air conditioned room $\left(22^{\circ} \mathrm{C}\right)$ with 12 hour cycle of light and dark. They had free access to water and the diets.

\section{Preparation of erythrocytes}

The rats were deprived of food for 14 to 16 hours, and, after ether anesthesia, blood was collected into tube containing EDTA by cardiac puncture. Blood plasma was separated by centrifugation at $1000 \mathrm{x}$ g for $5 \mathrm{~min}$. Erythrocytes were prepared after the collection of plasma. After removing the buffy coats and small portion of top layer of erythrocytes, the erythrocytes were washed three times in cold isotonic saline at $1000 \mathrm{~g}$ for $5 \mathrm{~min}$ at $4^{\circ} \mathrm{C}$. A small portion of the top layer was removed at each washing.

\section{Biochemical analysis of plasma}

A part of the plasma was sent to a clinical laboratory center (CRC Co, Fukuoka, Japan) and routine clinical laboratory tests of plasma including plasma phosholipid were done using an automated analyzer (Olympus AU-

Table 7 Change in erythrocyte phospholipids of Wistar rats after the PIsEtn diet for 9 weeks

\begin{tabular}{lccc}
\hline & Control diet $(\mathbf{n}=\mathbf{8})$ & PlsEtn diet $(\mathbf{n}=\mathbf{8})$ & t-test \\
\hline PlsEtn & $15.76 \pm 0.78$ & $16.61 \pm 0.83$ & $p<0.03$ \\
\hline PE & $8.45 \pm 0.71$ & $8.35 \pm 0.49$ & \\
\hline PlsCho & $0.52 \pm 0.12$ & $0.51 \pm 0.09$ & \\
\hline PC & $57.91 \pm 2.04$ & $56.71 \pm 0.91$ & $p<0.08$ \\
\hline PS & $4.81 \pm 0.92$ & $4.91 \pm 0.99$ & \\
\hline PI & $1.11 \pm 0.21$ & $1.21 \pm 0.37$ & \\
\hline SM & $10.32 \pm 1.09$ & $10.64 \pm 0.62$ & \\
\hline
\end{tabular}

Data are \% of total phospholipids calculated from chromatographic area detected by ELSD (mean \pm SD).
Table 8 Fatty acid composition of erythrocyte phospholipids of Wistar rats after the PIsEtn diet for 9 weeks

\begin{tabular}{llll}
\hline Fatty acid & Control diet & PlsEtn diet & t-test \\
\hline \multicolumn{4}{l}{ [A] Fatty acid composition of erythrocyte PIsEtn + PE } \\
\hline $22: 6+14: 0$ & $5.46 \pm 0.52$ & $5.70 \pm 0.38$ & \\
\hline $20: 4$ & $30.67 \pm 4.89$ & $35.06 \pm 2.91$ & $p<0.05$ \\
\hline $18: 2$ & $10.21 \pm 1.26$ & $10.33 \pm 0.50$ & \\
\hline $18: 1$ & $18.53 \pm 2.29$ & $17.69 \pm 1.94$ & \\
\hline $16: 0$ & $23.17 \pm 2.68$ & $20.87 \pm 1.60$ & $p<0.06$ \\
\hline $18: 0$ & $10.05 \pm 2.13$ & $8.02 \pm 1.49$ & $p<0.05$ \\
\hline other & $1.92 \pm 0.44$ & $2.33 \pm 0.81$ & \\
\hline
\end{tabular}

[B] Fatty acid composition of erythrocyte PC

\begin{tabular}{llll}
\hline $22: 6+14: 0$ & $2.32 \pm 0.41$ & $2.41 \pm 0.32$ & \\
\hline $20: 4$ & $8.63 \pm 2.89$ & $11.01 \pm 1.71$ & $p<0.07$ \\
\hline $18: 2$ & $10.12 \pm 1.12$ & $11.76 \pm 0.94$ & \\
\hline $18: 1$ & $11.63 \pm 0.73$ & $11.58 \pm 0.47$ & \\
\hline $16: 0$ & $48.81 \pm 3.44$ & $45.64 \pm 3.07$ & $p<0.07$ \\
\hline $18: 0$ & $16.21 \pm 1.08$ & $15.33 \pm 1.30$ & \\
\hline other & $2.28 \pm 0.41$ & $2.29 \pm 0.47$ & \\
\hline
\end{tabular}

Data are $\%$ of total fatty acids (mean \pm SD).

5200), in which total cholesterol, total phospholipid and triacylglycerol were determined by each enzymatic method (Cholsterol-C test, Phospholipid-B test and Triglyceride-G test, Wako Pure Chemical Co Osaka, Japan).

\section{Extraction of total lipids}

Extraction of total lipids from erythrocytes was done immediately after the preparation of the washed erythrocytes $[8,23,24]$. Briefly $500 \mu \mathrm{L}$ of the packed erythrocytes was hemolyzed with equal volume of water. Four $\mathrm{mL}$ of methanol was added to lysate followed after $40 \mathrm{~min}$ by $4 \mathrm{~mL}$ chloroform. After an additional $30 \mathrm{~min}$, the extract was centrifuged and residue was re-extracted with $4 \mathrm{~mL}$ of chloroform/methanol (1:1). Pooled extracts were washed with $10 \mathrm{~mL} 0.88 \% \mathrm{KCl}$ to make biphasic mixture. Two $\mathrm{mL}$ of lower phase was dried under N2 gas. Extraction of total lipid from plasma $(500 \mu \mathrm{L})$ was also accorded to that from the erythrocytes. Methanol and chloroform used in the extraction of lipids contained butylhydroxytoluene $(50 \mathrm{mg} / \mathrm{L})$.

\section{Separation of phospholipid classes}

Separation of phospholipid classes including plasmalogens (Pls) was done by our reported method [8]. The HPLC system used was an Agilent HPLC system (HP1100 Series, Agilent Technologies, Tokyo, Japan) equipped with an evaporative light scattering detector (ELSD), a fluorescence detector and a UV dector. The 
system was connected to a Chem Station (Agilent Technology) for control and analysis of chromatograms. Relative composition of phospholipid classes were measured on each chromatographic area with ELSD detection.

\section{Analysis of fatty acid composition of phospholipids}

Each phospholipid was collected from an HPLC with UV detection [23,24], and analysis of fatty acid composition of phospholipids was accorded to our previous reports $[23,24]$.

\section{Data analysis}

Data were analyzed by using a paired $t$ test (two tailed) with $\mathrm{p}<0.05$ used for significance.

\section{Competing interests}

The authors declare that they have no competing interests.

\section{Authors' contributions}

SM operated all experiments and wrote the manuscript.TK participated in critical discussion. KM prepared the purified plasmalogens. TF designed the study and participated in critical discussion. All authors read and approved the final manuscript

\section{Acknowledgements}

This work was supported in part by trust cost of The National Agriculture and Food Research Organization of Japan.

\section{Author details}

'Institute of Rheological Function of Food, 2241 Kubara, Hisayama-chou, Kasuya-gun, Fukuoka 811-2501, Japan. ²Department of Integrative Physiology, Graduate School of Medical Science Kyushu University, Fukuoka 812-8582, Japan. ${ }^{3}$ Central Research Institute, Marudai Food Co. Ltd, Osaka, Japan. ${ }^{4}$ Institute of Rheological Function of Food, 2241 Kubara, Hisayama-chou, Kasuya-gun, Fukuoka 811-2501, Japan.

Received: 5 September 2012 Accepted: 18 November 2012 Published: 21 November 2012

\section{References}

1. Farooqui AA, Horrocks LA: Plasmalogens: workhorse lipids of membranes in normal and injured neurons and glia. Neuroscientist 2001, 7:232-245.

2. Brites P, Waterham HR, Wanders RJA: Functions and biosynthesis of plasmalogens in health and disease. Biochim Biophys Acta 2004, 1636:219-231.

3. Wallner S, Schmitz G: Plasmalogens the neglected regulatory and scavenging lipid species. Chemistry Physics Lipids 2011, 164:573-589.

4. Braverman NE, Moser AB: Functions of plasmalogen lipids in health and disease. Biochim Biophys Acta 2012, 1822:1442-1452.

5. da Silva TF, Sousa VF, Malheiro AR, Brites P: The importance of ether-phospholipids: A view from the perspective of mouse models. Biochim Biophys Acta 2012, 1822:1501-1508.

6. Kullenberg D, Taylor LA, Schneider M, Massing U: Health effects of dietary phospholipids. Lipids Health Dis 2012, 11:3.

7. Murphy EJ, Stephens R, Jurkowitz-Alexander M, Horrocks LA: Acidic hydrolysis of plasmalogens followed by high-performance liquid chromatography. Lipids 1993, 28:565-568.

8. Mawatari S, Okuma Y, Fujino T: Separation of intact plasmalogens and all other phospholipids by a single run of high-performance liquid chromatography. Anal Biochem 2007, 370:54-59.

9. Cohn JS, Kamili A, Wat E, Chung RW, Tandy S: Dietary phospholipids and intestinal cholesterol absorption. Nutrients 2010, 2:116-127.

10. Shirouchi B, Nagao K, Furuya K, Inoue N, Inafuku M, Nasu M, Otsubo K, Koga $\mathrm{S}$, Matsumoto $\mathrm{H}$, Yanagita T: Effects of dietary phosphatidylinositol on cholesterol metabolism in Zucker (fa/fa) rats. J Oleo Sci 2009, 58:111-115.
11. Imaizumi K, Mawatari K, Murata M, Ikeda I, Sugano M: The contrasting effect of dietary phosphatidylethanolamine and phosphatidylcholine on plasma lipoproteins and liver lipids in rats. J Nutr 1983, 113:2403-2411.

12. Zierenberg $\mathrm{O}$, Grundy SM: Intestinal absorption of polyenephosphatidylcholine in man. J Lipid Res 1982, 23:1136-1142.

13. Jurkowitz MS, Horrocks LA, Litsky ML: Identification and characterization of alkenyl hydrolase (lysoplasmalogenase in microsomes and identification of a plasmalogen-active phospholipase $A 2$ in cytosol of small intestinal epithelium. Biochim Biophys Acta 1999, 1437:142-156.

14. Magnusson CD, Haraldsson GG: Ether lipids. Chem Phys Lipids 2011 $164: 315-340$

15. Das AK, Holmes RD, WIson GN, Hajra AK: Dietary ether lipid incorporation into tissue plasmalogens of humans and rodents. Lipids 1992, 27:401-405.

16. Das AK, Hajra AK: High incorporation of dietary 1-0-heptadecyl glycerol into tissue plasmalogens of young rats. FEBS Lett 1988, 227:187-190.

17. Brites P, Ferreira AS, Da Silva TF, Sousa VF, Malheiro AR, Duran M, Waterham HR, Baes M, Wanders RJ: Alkyl-glycerol rescues plasmalogen levels and pathology of ether-phospholipid deficient mice. PLoS One 2011, 6:e28539.

18. Blank ML, Cress EA, Smith ZL, Snyder F: Dietary supplementation with ether-linked lipids and tissue lipid composition. Lipids 1991, 26:166-169.

19. Nishimukai M, Wakisaka T, Hara H: Ingestion of plasmalogen markedly increased plasmalogen levels of blood plasma in rats. Lipids 2003, 38:1227-1235

20. Wood PL, Khan MA, Smith T, Ehrmantraut G, Jin W, Cui W, Braverman NE, Goodenowe DB: In vitro and in vivo plasmalogen replacement evaluations in rhizomelic chrondrodysplasia punctata and Pelizaeus-Merzbacher disease using PPI-1011, an ether lipids plasmalogen precursor. Lipid Health Dis 2011, 10:182.

21. Wood PL, Smith T, Lane N, Khan MA, Ehrmantraut G, Goodenowe DB: Oral bioavailability of the ether lipid plasmalogen precursor, PPI-1011, in the rabbit: a new therapeutic strategy for Alzheimer's disease. Lipids Health Dis 2011, 10:227.

22. Katafuchi T, Ifuku M, Mawatari S, Noda M, Miake K, Sugiyama M, Fujino T: Effects of plasmalogens on systemic lipopolysaccharide-induced glial activation and ß-amyloid accumulation in adult mice. Ann NY Acad SC 2012, 1262:85-92.

23. Mawatari S, Yunoki K, Sugiyama M, Fujino T: Simultaneous preparation of purified plasmalogens and sphingomyelin in human erythrocytes with phospholipase A1 from Aspergillus orizae. Biosci Biotechnol Biochem 2009, 73:2621-2625

24. Mawatari S, Murakami K: Analysis of membrane phospholipid peroxidation by isocratic high-performance liquid chromatography with ultraviolet detection. Anal Biochem 1998, 264:118-123.

doi:10.1186/1476-511X-11-161

Cite this article as: Mawatari et al:: Dietary plasmalogen increases erythrocyte membrane plasmalogen in rats. Lipids in Health and Disease 2012 11:161.

\section{Submit your next manuscript to BioMed Central and take full advantage of:}

- Convenient online submission

- Thorough peer review

- No space constraints or color figure charges

- Immediate publication on acceptance

- Inclusion in PubMed, CAS, Scopus and Google Scholar

- Research which is freely available for redistribution 